\title{
Sample preparation optimization for the simultaneous determination of mycotoxins in cereals.
}

\begin{abstract}
The efficiency of three extraction solvents and three clean-up procedures was compared for simultaneous extraction and purification of aflatoxins (AFB1, AFB2, AFG1 and AFG2), ochratoxin A (OTA), and zearalenone (ZEA) from spiked cereal samples. The best recovery rates for all mycotoxins were achieved using methanol: water (80:20) as the extraction solvent and AOZ multi-functional immunoaffinity column (IAC), as clean up method with recovery values of 61-89\%, while that of Oasis HLB and MycoSep 226 were 37-67\% and 44$78 \%$, respectively. Then, five variables in the IAC clean-up conditions, including primary conditioning with phosphate buffer saline (PBS) (0-10 mL) (X1), extract load up volume (10$20 \mathrm{~mL}$ ) (X2), washing volume with PBS (10-20 mL) (X3), and eluting solution volumes with methanol (1-3 mL) (X4) and acetic acid (0-1.5 mL) (X5), were optimized for the specific purification and enrichment of the mycotoxins. Results showed that primary conditioning and PBS washing did not have a significant effect on the recovery responses of mycotoxins. Optimized conditions were selected as $0,15,10,1.3$, and $1.5 \mathrm{~mL}$ for $\mathrm{X} 1-\mathrm{X} 5$, respectively. The recovery rates of AFB1, AFB2, AFG1, AFG2, OTA and ZEA were within $93-104 \%$ in spiked rice, under optimal conditions. LOD and LOQ were 0.0125 and $0.05 \mathrm{ng} / \mathrm{g}$ for AFB1 and AFG1, 0.0037 and $0.015 \mathrm{ng} / \mathrm{g}$ for AFB2 and AFG2, 0.05 and $0.2 \mathrm{ng} / \mathrm{g}$ for OTA, and 0.5 and $2 \mathrm{ng} / \mathrm{g}$ for ZEA, respectively. Extraction of spiked cereal samples with methanol: water (80:20) and clean up using AOZ IAC column in optimal condition provided recovery range of $77-104 \%$ for all targeted mycotoxins.
\end{abstract}

Keyword: Sample preparation; Optimization; Mycotoxin; Cereal; HPLC. 